\title{
Recent advances in the formation of phase inversion membranes made from amorphous or semi-crystalline polymers
}

\author{
I.M. Wienk, R.M Boom, M.A.M. Beerlage, A.M.W. Bulte, C.A. Smolders, \\ H. Strathmann \\ University of Twente, P.O. Box 217, 7500 AE Enschede, Netherlands
}

Accepted 11 September 1995

\begin{abstract}
Structural characteristics in membranes formed by diffusion induced phase separation processes are discussed. Established theories on membrane formation from ternary systems can be extended to describe the effects of high or low molecular weight additives. A mechanism for the formation of nodular structures in the top layer of ultrafiltration membranes is presented. In the last part structures arising from polymer crystallization during immersion precipitation are discussed.
\end{abstract}

Keywords: Membrane formation; Additives; Macrovoids; Nodules; Crystallizable polymers

\section{Introduction}

For more than thirty years structural characteristics of phase inversion membranes from amorphous polymers have attained much attention. Problems to be solved in a semi-quantitative way were: the absence or presence of a skin layer and its porosity, the occurrence of macrovoids and of nodular structures. A breakthrough in understanding the origin of these structural elements was obtained by:

(i) thermodynamic calculation of 3-component phase diagrams (polymer/solvent/non-solvent), using proper binary interaction parameters, in combination with

(ii) computations of so-called composition paths for tracing the kinetics of phase separation, using proper diffusion equations, boundary conditions and frictional parameters both for the polymer film and the coagulation bath.
Recent attention in research on membrane formation at the University of Twente has focused on:

- the role of additives, both high- and low-molecular weight, on membrane formation

- further studies on macrovoid formation

- the background of formation of nodular structures.

Apart from these studies on systems with amorphous polymers, the above mentioned computational approach of phase separation and composition paths has been applied also to membrane formation using systems with a rapidly crystallizable polymer.

\section{Membrane formation: state of the art}

\subsection{Phase diagrams}

The structure of phase inversion membranes results from a phase change of initially stable solutions 
which have been brought to an unstable state. The state and equilibrium compositions of polymer solutions can be well depicted in ternary phase diagrams. In the metastable region, lying between the binodal and spinodal curves, phase separation will take place by nucleation and growth. In the unstable region of the phase diagram spinodal decomposition will occur. During nucleation in membrane forming systems the polymer lean phase will nucleate and the polymer rich phase will be continuous. The concentration of the polymer rich phase increases until locally the glass transition of the system is reached and vitrification of the solution takes place. Once the polymer rich phase has attained a certain rigidity profound changes in morphology are not possible any more. The ultimate structure of this process is a porous membrane [1].

The location of the binodal curve in the ternary phase diagram, enclosing the demixing gap, can be found by cloud-point measurements or by measuring the light scattering of the polymer solutions for varying compositions [2], or it can be calculated if the thermodynamic interaction parameters of the system are known. Tompa [3] and Altena et al. [4] calculated phase diagrams for ternary systems of amorphous polymers using binary interaction parameters.

\subsection{Composition paths}

Phase separation of a polymer solution can be induced by:

- quenching the solution to a lower temperature

- immersion of the solution in a bath of non-solvent

- contacting the polymer solution with a vapour of the non-solvent

- evaporation of the solvent.

For membrane formation immersion precipitation is the most important and best studied process. Phase separation processes by evaporation or contact with a non-solvent vapour are closely related to the immersion precipitation process because they are also diffusion induced.

Upon immersion of a film of a polymer solution into a non-solvent or coagulation bath the non-solvent will diffuse into the polymer solution, whereas the solvent diffuses into the bath. The diffusion coefficient of the polymer is much lower and therefore the mobility of these polymeric molecules will reach only for smaller distances.

Fundamental studies on membrane formation by immersion precipitation in ternary systems of polymer, solvent and non-solvent have been carried out by Cohen et al. [5] Reuvers et al. [6], Tsay et al. [7] and Radovanovic et al. [8]. They developed mass transfer models to describe the diffusion processes for especially the first moments of immersion. Such a kinetic model results in a composition path in the ternary phase diagram. This path represents either the change in composition of a small volume in time or the compositions at all loci in the film at a certain moment. Combined with the equilibrium thermodynamics of the system it can be determined when and where the composition path will cross the binodal curve. So it is possible to predict at what time after immersion phase separation will start near the interface and what the composition profile of the polymer solution will be at that moment.

Based on the calculated composition paths and supported by experimental data Reuvers et al. [6] divided membrane formation processes into two groups: instantaneous demixing systems and systems that show delay of demixing. If for a given system (a thin solution film) at a short moment after immersion the composition path crosses the binodal the process is called instantaneous demixing. For systems that have a delay time of demixing the composition path will not cross the binodal curve until that (delay) time has passed. Membranes formed by instantaneous demixing generally show a highly porous substructure (with macrovoids) and a finely porous, thin skin layer. Membranes formed by a delayed demixing mechanism show a porous (often closed-cell, macrovoid-free) substructure with a dense, relatively thick skin layer. Which process takes place is mainly determined by the interaction between the solvent and the non-solvent, and by the non-solvent concentration in the bath.

Very often large conical voids, called macrovoids are observed in diffusion induced phase separation membranes. The mechanism of macrovoid formation has long been a controversial subject. Macrovoids are thought to be initiated by surface tension gradients (Matz [9], Frommer [10], Stevens [11]) or mechanical stresses (Strathmann [12], Craig [13]). Gröbe [14] and Broens et al. [15] suggested that macrovoids 
are formed by anomalous growth of nuclei, because the influx of non-solvent is so small, that the growth of the macrovoid can keep pace with the phase separation induced by the non-solvent.

Smolders et al. [16] were able to relate the mechanism suggested by Gröbe to the mass transfer model by Reuvers. They reasoned that under the top layer, locally nuclei could be created that contain quite a high solvent concentration. This could induce local delay of demixing, which keeps the polymer solution around the nucleus stable while the nucleus is growing. As long as the solution remains stable, no new nuclei deeper in the membrane are formed and the nucleus can grow to form a macrovoid.

The mass transfer model is valid only for homogeneous polymer solutions (i.e. the trajects outside the demixing gap). As soon as the polymer solution demixes the mass exchange with the immersion bath will be different for the two phases. More information is necessary, than what is available now, on how the compositions and the volumes of the two phases change in time, to be able to expand the mass transfer model for the inhomogeneous region.

Despite its restrictions the mass transfer model is suitable to explain qualitatively the effects of system variables, such as concentration, temperature and interaction between the components, on the structure of the ultimate membrane.

\section{The role of a high molecular weight additive in membrane formation}

The addition of a high molecular weight component to the polymer solution changes drastically the structure of the membranes; the porosity is higher, pores are well interconnected and macrovoid formation is suppressed. The best known example of these systems is the use of poly(vinyl pyrrolidone), PVP, a water soluble polymer, in solutions of polysulfone, PSF, poly(ether sulfone), PES, or poly(ether imide), PEI, in $N$-methyl pyrrolidone, NMP.

Only a few authors have investigated the mechanism of membrane formation from a system with a high molecular weight additive. Cabasso [17] suggested that microphase demixing between the two polymers takes place which prevents the formation of the dense top layer. According to Roesink [18] the high interconnectivity of the pores in microfiltration membranes containing PEI and PVP is the result of thin PVP walls between the pores that break upon drying the membrane.

Boom et al. [19] studied the quaternary system PES/PVP/NMP/water. To find a mechanism that describes the formation of the characteristic structures of PVP-containing membranes a new approach of phase separation was followed. The interaction between PVP and PES is very good and the diffusion of the two polymers with respect to each other is slow. Therefore Boom regarded the two polymers at the very beginning of the mass exchange process as one constituent, having intermediate values for the interactions with NMP and water compared to those for PES and PVP separately. It was found that the demixing gap of such a (virtual) quasi ternary system is strongly reduced in magnitude; the virtual system can contain a lot of the non-solvent water (up to 50 volume \%) without demixing. In Fig. $1 \mathrm{~b}$ the so-called virtual binodals belonging to the quasi ternary system are shown for different ratios of PES and PVP. This situation is only valid for a very short time. When the two polymers are regarded as freely moving species the demixing gap is much more expanded and a small amount of water will cause demixing of the system (Fig. 1a). The binodal belonging to the quaternary system will be indicated as the equilibrium binodal. This binodal coincides with the cloudpoint curve of the system (see Fig. 1).

Boom et al. [19] defined two time scales for diffusion. One is a short time scale for the diffusion of solvent and non-solvent between polymer segments. On this scale the interdiffusion of the two polymers relative to each other is negligible. The system is quasi ternary and the virtual binodals are valid. On the other hand there is a long time scale for the diffusion of the two polymers with respect to each other. For the first moments (fractions of seconds) after immersion the phenomena taking place on a short time scale are the most important events. But as the diffusion processes proceed further the long time scale becomes more important.

With this model the effect of PVP as pore former can be explained. A composition path for immersion in the pure non-solvent as calculated by Boom et al. [19] is shown in Fig. 1a. For the calculation of this composition path, only the diffusion of solvent and 
non-solvent are considered and the virtual binodals are used. However immediately after immersion, the cloudpoint line is reached and the diffusion of the two polymers with respect to each other will start (although this diffusion takes place on the long time scale). Thus when the equilibrium binodal (cloud point line) is considered the realized composition path will lie inside the demixing gap indicating the occurrence of instantaneous demixing. In this way, the typical conditions for delayed demixing (composition path outside of the binodal curve) will essentially be excluded. As said before, instantaneously demixing systems lead to the formation of porous structures.

Boom et al. [19] calculated the equilibrium compositions of the two phases arising from phase separation of these quaternary systems. The calculations indicate that one phase consists of PES, NMP and water while the other consists of PVP, NMP and water. It seems that the two polymers have a driving force to separate completely into a PES-rich and a PVP-rich phase. Thus the demixing process of the system is mainly determined by the diffusion of the two polymers with respect to each other. The incubation time for nucleation is zero (as was indicated by light transmission measurements performed by Boom et al. [2]) thus demixing starts as soon as the equilibrium binodal is passed. However because the diffusional exchange of the solvent and the non-solvent with the coagulation medium is much faster than the demixing process there still can be a large supersaturation of the polymer solution. Such a situation differs essentially from immersion precipitation using a ternary system. In the latter case one could not pass the metastable region without formation of nuclei of the polymer-lean phase.

According to this mechanism the composition paths might lie even in the instable region. Therefore Boom et al. [19] showed that for these systems spinodal decomposition occurs which can be an explanation for the high interconnectivity of the pores in membranes containing PVP. In this case the interconnected pores are in fact a continuous polymer (PES) lean phase intertwined by a continuous polymer (PES) rich phase which forms the membrane matrix.

The presence of PVP is very effective in macrovoid suppression. According to the model of Smolders et al. [16] macrovoids can arise by growth of nuclei with a high solvent concentration. However for these quaternary systems the formation of nuclei having a PVP rich phase is so slow compared to the diffusion of non-solvent into the polymer solution that nuclei with a high solvent concentration are not present. Furthermore high supersaturation results in a highly unstable polymer solution whereas a condition for growth of macrovoids would be a stable polymer solution in front of it.

Phase separation causes the formation of a polymer (PES) rich and a polymer lean (PVP rich) phase.
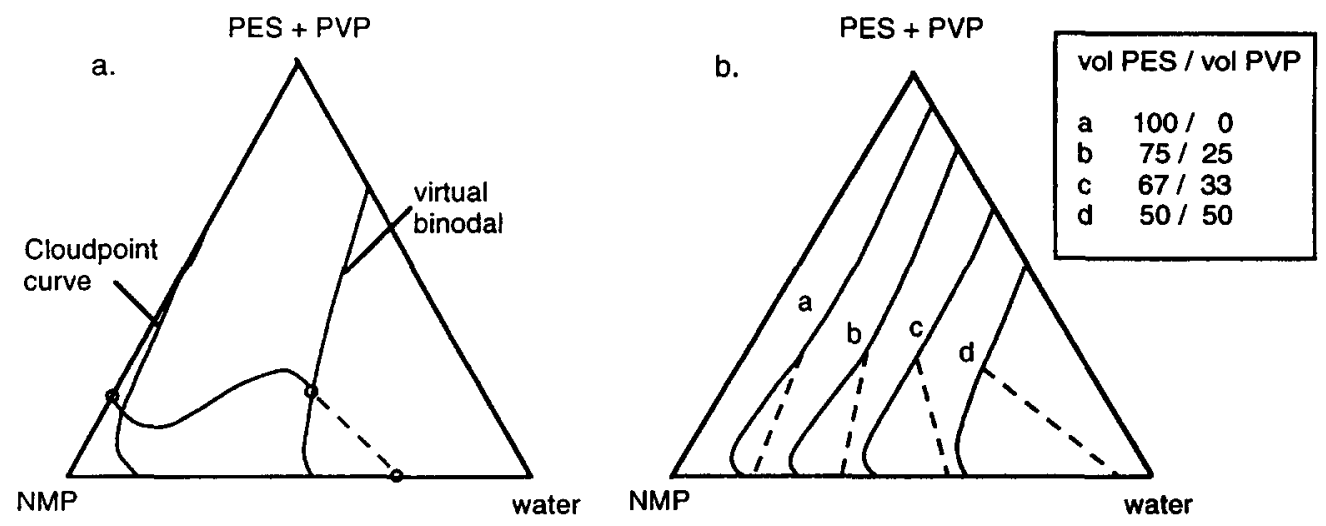

Fig. 1. Cross sections through quatemary phase diagrams at a constant ratio between the concentrations of PES and PVP. (a) Cloud point curve (equilibrium binodal) and virtual binodal for a system with an equal amount of PES and PVP. Also in this figure an initial composition path for the immersion of a solution containing $20 \mathrm{vol} \%$ polymer (PES and PVP) in water. (b) calculated virtual binodals for the system PES-PVP-NMP-water, valid as long as no movement between the two polymers is possible. Dashed lines are tie-lines. 
In the polymer rich phase the growth in concentration of PES will profoundly increase the viscosity of this phase until vitrification occurs which is generally considered as the end of the structure formation process. At the moment of vitrification the equilibrium composition has not yet been reached and part of the PVP molecules are permanently trapped in the PES matrix. The result of this entrapment is a membrane with a hydrophilic character, through the residual presence of a certain (small) amount of PVP molecules [19].

\section{The role of a low molecular weight additive on membrane formation}

Even more often than a second polymer, low molecular weight additives are used to improve the morphology and performance of membranes. Also for this type of additive the number of fundamental studies concerning its effect on membrane formation is limited. Usually the influence of a low molecular weight additive is ascribed to specific interaction with the polymer resulting in swelling of the polymer (Keilin [20] and King et al. [21]) or the formation of supermolecular aggregates (Kesting et al. [22], Kunst et al. [23], Vásárhelyi et al. [24] and Ronner et al. [25]). Kesting et al. [22b] also describes an effect of the addition of a carboxylic acid (nonsolvent for the polymer) on the flux properties (hence the skin thickness) of gas separation membranes. They describe the association of the additive with the solvent used (DMF, NMP). Since the explanation is focused on flux improvement of already void-less membranes, it does not give a genuine explanation for prevention of macrovoid formation.

Recently Beerlage [26] used a number of dicarboxylic acids as additives in polyimide/dimethylformamide solutions for the preparation of ultrafiltration membranes. It was found that some of the dicarboxylic acids reduce the number of pores in the top layer and suppress the formation of macrovoids; the best results were obtained using oxalic acid. Beerlage found that in order to be effective the dicarboxylic acid has to meet three criteria:

- at least two approachable $-\mathrm{OH}$ groups have to be present

- the solubility of the acid in the coagulation bath has to be low

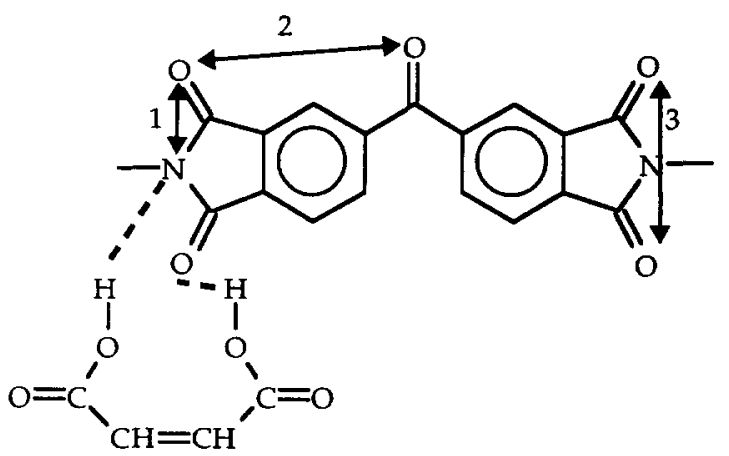

Fig. 2. Schematic drawing of the complex formation of maleic acid and polyimide. The complex formation results in decreased flexibility of the polyimide chain since the imide nitrogen atom serves as a pivot-point, i.e. a flexible link.

- intramolecular hydrogen bonding in the additive molecule should not be possible.

Effective dicarboxylic acid molecules are able to form with their two - $\mathrm{OH}$ groups a bridge-complex on the imide carbon:l and nitrogen (Fig. 2). As was shown by Smit [27] the imide nitrogen atom is a flexible link or "pivot point" of the polyimide chain. The complex formation thereby causes a decrease in polymer chain flexibility due to steric hindrance at the pivot point. Therefore, the result of complex formation may well be a change in the polymer properties, as was also indicated by a visible increase in polymer solution viscosity upon addition of an effective dicarboxylic acid.

Thus the dicarboxylic acid causes temporarily a decrease of polymer chain mobility. The stability of the acid polymer complex is higher in case the interaction between acid and non-solvent is lower i.e. when the solubility of the dicarboxylic acid in the coagulation bath is lower. Knowing this, parallels of this system with the quaternary system containing a macromolecular additive can be drawn.

According to the mechanism of Boom two time scales can be defined. A short time scale for the diffusion of solvent and non-solvent. On this scale the polymer chain behaves as a complex with the dicarboxylic acid resulting in a changed interaction with solvent and non-solvent and a reduced mobility (comparable to the idea that PVP and PES can be regarded as one constituent). The second time scale is a long time scale for breaking up the bridge-complex and removal of the dicarboxylic acid (compara- 
ble to the diffusion of PES and PVP with respect to each other). Boom found that the two time scales result in differences in membrane structure only if the polymer-polymer diffusion is slow, i.e. if the molecular weight of the additive is high. For the system of Beerlage the long time scale can only be effective if the complex bonding is strong, i.e. if the interaction between the dicarboxylic acid and polymer is high compared to the interaction between the acid group and the non-solvent.

Because of the similarity of the systems with a low and a high molecular weight additive, the mechanism of macrovoid suppression for the system studied by Beerlage is assumed to be equal to the mechanism described by Boom, i.e. the continuous creation of instability conditions ahead of the nucleation front.

Two remarks have to be made here. Boom showed that in the system of study one constituent of "PES/PVP" has a very small (virtual) demixing gap compared to the quaternary system. In the system of Beerlage the demixing gap determined by cloud point measurements is larger in case the additive is present but it is not certain (yet) that the polymer acid complex has a smaller virtual demixing gap. Secondly the addition of a macromolecular component causes an increase of surface porosity whereas Beerlage found a decrease of the number of pores. The latter was explained by inability of the stiff polymer chains, resulting from the presence of the acid group at the pivot point, to rapidly disentangle which is necessary for the creation of pores.

\section{The formation of nodular structures in mem- brane top layers}

Very rapid precipitation conditions during membrane formation, i.e. fast exchange of solvent and non-solvent, may result in a membrane top layer consisting of closely packed spheres or nodules. A nodular structure is often present in ultrafiltration membranes and sometimes in gas separation membranes as well.

In literature the origin of the nodules has been ascribed to micelles (Panar et al. [28]), para-crystallites (Kesting [29]), aggregates (Kamide et al. [30]), inhomogeneities in the polymer solution (Kunst et al.
[31]) or perturbations (Ray et al. [32]). More recently Kimmerle et al. [33] proposed that the spheres are formed by nucleation of a polymer rich phase. Pinnau et al. [34] and Boom et al. [35] state that the diffusion processes during formation of the top layer are fast enough for the polymer solution to become highly instable and cross the spinodal curve. In this mechanism nodule formation is supposed to result from spinodal decomposition.

Two aspects of nodule formation are definitely established: (i) the diffusion processes of solvent and non-solvent are very rapid, especially compared to the mobility of the polymer molecules and (ii) nodule formation occurs in a thin $(<1.0 \mu \mathrm{m})$ surface layer of very high polymer concentration (above 90\%).

Because of the enormous increase of polymer concentration in the top layer nucleation of a polymer rich phase is not likely to take place. It is even so that the binodal and spinodal curve have lost their signification. The thermodynamic data of the binodal and spinodal curves are based on equilibrium conditions. If the kinetics of the process are very rapid, equilibrium thermodynamics will not give adequate information on the compositions in the demixing system at the time and place of inspection. It is therefore better to abandon the equilibrium phase diagram and study this process on a molecular level.

Recently Wienk et al. [36] described a new mechanism for the formation of nodular structures. Due to rapid out-diffusion of the solvent and simultaneous indiffusion of the non-solvent, the polymer molecules are confronted with a non-compatible environment. In reaction with this sudden change of state the polymer molecules surround themselves with other polymer segments. In dilute polymer solutions it is often observed that a polymer coil collapses if the solubility strength of the environment is reduced by, e.g., a temperature quench [37]. In the systems discussed here the situation is more complicated. Due to the high concentration the polymer chains are not extended but are highly entangled coils. The collapse of a molecule in a polymer network should not be compared with a rather slow repetition diffusion process. The polymer molecules can diminish their interactions with the non-solvent also by clustering of polymer segments into groups, which is much faster. These polymer clusters may consist of seg- 
ments of different molecules, whereas adjacent clusters are connected by entanglements and by shared polymer chains. The size of each cluster might be determined by conditions of spinodal decomposition.

In the beginning of this process the clusters are highly connected because some polymer molecules are entangled in adjacent clusters. In a later stage disentanglement of polymer segments between the spheres may lead to a pore formation process. However, excess out-diffusion of solvent increases the polymer concentration of the top layer and therefore results in vitrification of the polymer matrix. The centres of the polymer clusters are the first to reach the concentration at which vitrification occurs. Hence, disentanglement and vitrification occur simultaneously. However, the very fast vitrification prevents the disentanglement process to be completed. In some cases (Pinnau et al. [34]) pore formation can even be prevented and a gas separation membrane results.

Beerlage [38] performed experiments that support this mechanism. It was observed that drying of polyimide ultrafiltration membranes prepared from polyimide solutions in DMF or DMAc resulted in a surface shrinkage of $18 \%$. Beerlage found that the surface shrinkage was:

- independent of the drying procedure

- independent of the initial hydraulic permeability of the membranes

- much higher than the wet state surface porosity of the membranes which was about $1 \%$.

On the other hand the surface shrinkage was higher (22\%) for membranes prepared from a polyimide solution in NMP.
From these results it was concluded that even though it is generally assumed that surface shrinkage is directly caused by pore collapse, there must be an even more important mechanism next to pore collapse that determines the shrinkage. Beerlage suggested that during membrane preparation strong tensions tend to be frozen in, and that liquid exchange and drying creates enough free volume inside the nodules in the top layer to relax these tensions.

Combining Wienk's mechanism for nodule formation with the results from Beerlage it is clear that the collapse of polymer molecules during formation of the top layer takes place mainly in the direction perpendicular to the surface. It is in this (normal) direction that an enormous densification of the polymer solution takes place in order to reach a layer with a polymer concentration above $90 \%$. This onedimensional collapse into a "flat" conformation of the molecules in the nodules causes large tensions in the other two dimensions, as well as voids inside the nodules. The voids are filled with residual solvent which is exchanged by non-solvent during rinsing of the membrane. Removal of the non-solvent in the drying procedure gives the flat nodules the opportunity for stress relaxation. The flat molecular conformations rearrange into spherical conformations, and a large surface shrinkage is the result. A schematic representation of this mechanism is shown in Fig. 3.

\section{Membrane formation using a rapidly crystalliz- able polymer}

A large research effort on membrane formation is dedicated to amorphous polymers. Often these theo-

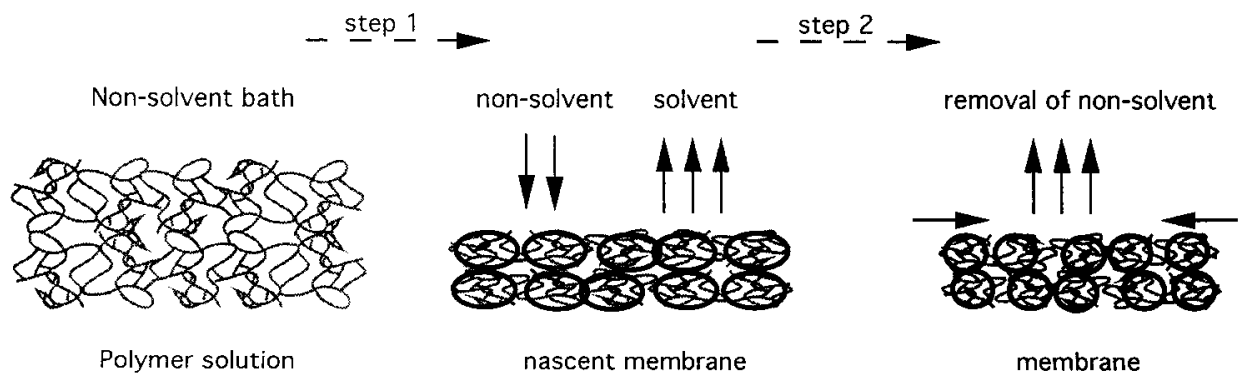

Fig. 3. Schematic representation of polymer collapse in the top layer of nascent ultrafiltration membranes. Step 1 shows the one-dimensional shrinkage of polymer molecules as response to the sudden non-compatible environment and increased polymer concentration. Step 2 shows the stress relaxation process resulting in spherical polymer nodules. 


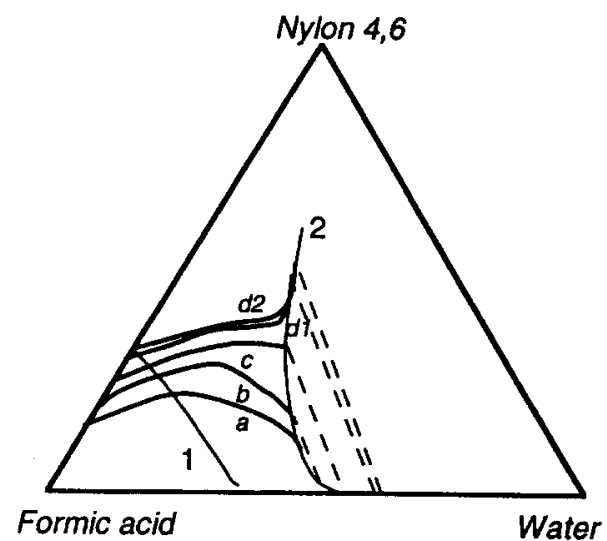

Fig. 4. Calculated composition paths for immersion of a film with initial polymer concentration of a: $15 \%, b: 20 \%$, c: $25 \%$, d1: $30 \%$ and $\mathrm{d} 2: 32 \%$. The experimental crystallization line (1) and the calculated binodal (2) are given.

ries are incorrectly used to describe the formation of membranes from semi-crystalline polymers. A fundamental study on polymer crystallization during immersion precipitation has been performed by Bulte [39]. The ternary system nylon-4,6, formic acid and water was used as a model system.

Membranes prepared by means of immersion precipitation with semi-crystalline polymers such as aliphatic nylons, show two types of phase separation processes: liquid-liquid demixing resulting in a porous structure with pores that are formed from the nucleated polymer lean phase and solid-liquid demixing with crystalline parts in the membrane. The pores in this case are the voids between the partly crystalline units.

Bulte worked out the equilibrium thermodynamics of the system. The binodal was calculated by means of the modified Flory-Huggins theory using proper binary interaction parameters. The isothermal crystallization line at $30^{\circ} \mathrm{C}$ was determined by extrapolation

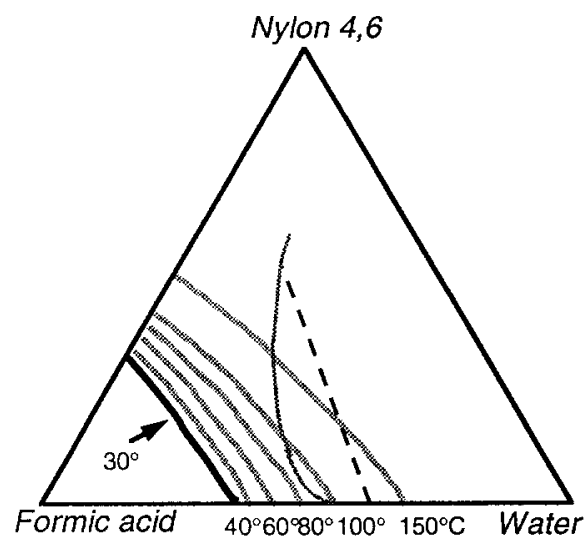

Fig. 5. Calculated (virtual) isotherms for the crystallization lines at various temperatures derived from experimentally derived melting points and the binodal curve at $30^{\circ} \mathrm{C}$ for the ternary system nylon-4,6, formic acid and water.

of experimentally derived melting points of ternary mixtures between 35 and $85^{\circ} \mathrm{C}$. The binodal is located inside the crystallization area which means that solid-liquid demixing is thermodynamically more favourable. However, crystallization can only be competitive to liquid-liquid demixing if the polymer solution has a certain supersaturation.

The diffusion induced degree of supersaturation is the main factor determining the type of membrane structure that will be obtained. To acquire information on the supersaturation Bulte assumed that for the first moments of immersion the binodal curve is valid only. Using the mass transfer model of Reuvers et al. [6], composition paths can be calculated that indicate the changes of composition in the polymer solution until the moment of phase separation (see Fig. 4). The diffusion induced degree of supersaturation for crystallization of the polymer can be expressed in terms of a chemical potential difference.

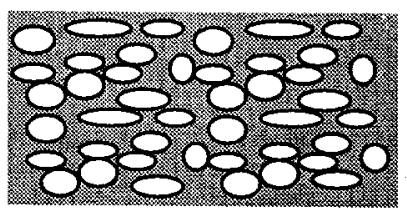

a.

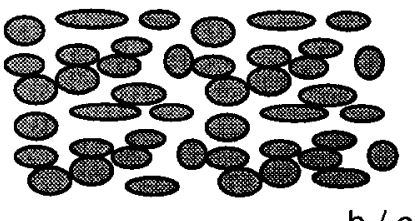

b / c.

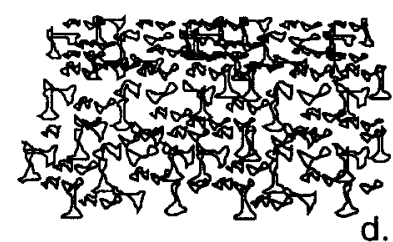

d.

Fig. 6. The three types of corresponding membrane morphologies referring to the situations in Fig. 4: "a" a cellular morphology resulting from liquid-liquid demixing, the white areas are open pores; " $b$ " and " $c$ " a spherulitic and " $d$ " an axialitic structure, resulting from solid-liquid phase separation with spherulitic crystallization of the polymer. The spherulite size in case " $b$ " is $10 \mu \mathrm{m}$ and in case " $c$ " 2 $\mu \mathrm{m}$. 
More insight can be obtained if this membrane formation process is compared to crystallization from the melt. To do so virtual melting isotherms for the crystallization lines at various temperatures can be calculated from experimentally derived melting points (see Fig. 5). If the composition of the polymer solution is located at a (virtual) crystallization line of higher temperature, it means that the undercooling, and thus the supersaturation is higher.

Comparing the cases " $a$ ", " $b$ ", " $c$ "' and " $d$ " in Fig. 4 it can be deduced that the degree of diffusion induced supersaturation increases from " $a$ " to " $d$ ". The related types of morphology are schematically depicted in Fig. 6. It can be concluded that the larger the degree of undercooling the finer is the membrane structure. The types of mechanisms, delayed liquidliquid demixing and solid-liquid demixing with an induction time, lead in most cases to membranes with a morphology controlled by crystallization. The nucleation of crystalline material is highly dependent on the degree of supersaturation. Smaller spherulite size is a result of a larger nucleation density resulting from a larger degree of supersaturation. If the supersaturation is too low, crystallization of the polymer takes place after the cellular morphology has formed (case "a"); nuclei of a crystalline phase could not be created on a short time scale. This type of morphology then is the result of liquid-liquid phase separation.

\section{Final remarks}

Membrane formation is a challenging field of research with still many unexplored aspects. Based on equilibrium thermodynamics and models of mass transfer, membrane formation mechanisms can be derived that are useful in describing more complex systems.

Recent advances in research at the University of Twente has given more insight in membrane formation from ternary systems with a rapidly crystallizable polymer and from quaternary systems with a high or a low molecular weight additive. Also the origin of typical membrane morphologies such as macrovoids and nodular top layers can be described as a result of fundamental research.

\section{References}

[1] (a) For binary systems: J. Arnauts and H. Berghmans, Amorphous thermoreversible gels of atactic polystyrene, Polymer Comm., 28 (1987) 66; R.M. Hikmet et al., Thermoreversible gelation of atactic polystyrene: phase transformation and morphology, Polymer, 29 (1988) 1378; Callister et al., Makrom. Chem. Makrom. Symp., 39 (1990)19. (b) For ternary systems: K. Kimmerle, PhD thesis, Chapter 1, University of Stuttgart, Germany, 1988; G.E. Gaides and A.J. McHugh, Gelation in an amorphous polymer: a discussion of its relation to membrane formation, Polymer, 30 (1989) 2118; I. Pinnau, PhD thesis, Chapter 2, University of Texas, USA, 1991; S.-G. Li, Preparation of hollow fiber membranes from gas separation, Thesis University of Twente, The Netherlands, 1994.

[2] (a) R.M. Boom, Th. van den Boomgaard and C.A. Smolders, Metastable demixing phenomena by thermal quench experiments. I. Theory, J. Appl. Polym. Sci., submitted: (b) R.M. Boom, S. Rekveld, U. Cordilia, Th. van den Boomgaard and C.A. Smolders, Metastable demixing phenomena by thermal quench experiments. II. The systems PES-NMP-water and PES-NMP-PVP-water, J. Appl. Polym. Sci., submitted.

[3] H. Tompa, Polymer solutions, Butterworths, London, 1956, p. 82.

[4] F.W. Altena and C.A. Smolders, Calculation of liquid-liquid phase separation in a ternary system of a polymer in a mixture of a solvent and a nonsolvent, Macromolecules, 15 (1982) 1491 .

[5] C. Cohen, G.B. Tanny and S. Prager, Diffusion controlled formation of porous structures in ternary polymer systems, J. Polym. Sci., Polym. Phys. Ed., 17 (1979) 477.

[6] (a) A.J. Reuvers, J.W.A. v.d. Berg and C.A. Smolders, Formation of membranes by means of immersion precipitation. I. A model to describe mass transfer during immersion precipitation, J. Membrane Sci., 34 (1987) 45. (b) A.J. Reuvers and C.A. Smolders, Formation of membranes by means of immersion precipitation. II. The mechanism of formation of membranes prepared from the system cellulose acetate-acetone-water, J. Membrane Sci., 34 (1987) 67.

[7] C.S. Tsay and A.J. McHugh, Mass transfer modeling of asymmetric membrane formation by phase inversion, J. Polyn. Sci., Part B, Polym. Phys., 28 (1990) 1327.

[8] (a) P. Radovanovic, S.W. Thiel and S.T. Hwang, Formation of asymmetric polysulfone membranes by immersion precipitation. Part I: Modelling mass transport during gelation, J. Membrane Sci., 65 (1992) 213. (b) P. Radovanovic, S.W. Thiel and S.T. Hwang, Formation of asymmetric polysulfone membranes by immersion precipitation. Part II: The effects of casting solution and gelation bath compositions on membrane structure and skin formation, J. Membrane Sci., 65 (1992) 231.

[9] R. Matz, The structure of cellulose acetate membranes. I.The development of porous structures in anisotropic membranes, Desalination, 10 (1972) 1.

[10] M.A. Frommer and R.M. Messalem, Mechanism of membrane formation. VI. Convective flows and large void forma- 
tion during membrane precipitation, Ind. Eng. Chem. Prod. Res. Dev., 12 (1973) 328.

[11] W.E. Stevens, C.S. Dunn and C.A. Petty, Surface tension induced cavitation in polymeric membranes during gelation, Paper presented at 73rd AIChE Annual Meeting, Chicago, Illinois, 1980.

[12] H. Strathmann, K. Kock, P. Amar, R.W. Baker, The formation mechanism of asymmetric membranes, Desalination, 16 (1975) 179.

[13] J.P. Craig, J.P. Knudsen and V.F. Holland, Textile Res. J., 32 (1962) 435.

[14] V. Gröbe, G. Mann and G. Duwe, Ausbildung von Strukturen bei der Koagulation von Polyacrylnitrillösungen, Fasenforsch. Textiltechn., 17 (1966) 142.

[15] L. Broens, F.W. Altena, C.A. Smolders, Asymmetric membrane structures as a result of phase separation phenomena, Desalination, 32 (1980) 33.

[16] C.A. Smolders, A.J. Reuvers, R.M. Boom and I.M. Wienk, Microstructures in phase-inversion membranes Part 1. Formation of macrovoids, J. Membrane Sci., 73 (1992) 259.

[17] (a) I. Cabasso, E. Klein and J.K. Smith, Polysulfone hollow fibers. I. Spinning and properties, J. Appl. Polym. Sci., 20 (1976) 2377. (b) I. Cabasso, E. Klein and J.K. Smith, Polysulfone hollow fibers. II. Morphology, J. Appl. Polym. Sci., 21 (1977) 165.

[18] H.D.W. Roesink, Microfiltration, membrane development and module design, PhD thesis University of Twente, The Netherlands, 1989.

[19] (a) R.M. Boom, Th. van den Boomgaard and C.A. Smolders, Equilibrium thermodynamics of a quatemary membrane forming system with two polymers. I. Theory, Macromolecules, submitted. (b) R.M. Boom, H.W. Reinders, H.H.W. Rolevink, U. Cordilia, Th. van den Boomgaard and C.A. Smolders, Equilibrium thermodynamics of a quaternary membrane forming system with two polymers. II. Experimental, Macromolecules, submitted. (c) R.M. Boom, Th. van den Boomgaard and C.A. Smolders, Mass transfer and thermodynamics during immersion precipitation for a two polymer system: Evaluation with the system PES-PVP-NMPwater, J. Membrane Sci., submitted. (d) R.M. Boom, S. Zanic, Th. van den Boomgaard and C.A. Smolders, Membranes from PES and PVP: Membrane morphology and its relation to the formation mechanism, J. Membrane Sci., submitted. (e) R.M. Boom, H.H.W. Rolevink, Th. van den Boomgaard and C.A. Smolders, Membranes prepared from PES and PS: Comparison with the PES-PVP system, J. Membrane Sci., submitted.

[20] (a) B. Keilin, Office of Saline Water report no. 84, United States, Department of the Interior, 1964. (b) B. Keilin, The mechanism of desalination by reverse osmosis, Office of Saline Water report no. 117 , United States, Department of the Interior, 1964.

[21] (a) W.M. King and P.A. Cantor, Reverse osmosis and process and composition for manufacturing cellulose acetate membranes wherein the swelling agent is a di- or tri-basic aliphatic acid, US Pat., 3,673,084, 1972. (b) W.M. King, D.L. Hoernschemeyer and C.W. Saltonstall, Cellulose acetate blend membranes, in H.K. Lonsdale and H.E. Podall (Eds.), Reverse Osmosis Membrane Research, Plenum, New York, 1972, p. 131.

[22] (a) R.E. Kesting and A. Menefee, The role of formamide in the preparation of cellulose acetate membranes by the phase inversion process, Kolloid Z. Z. Polym., 230 (1969) 341. (b) R.E. Kesting and A.K. Fritzsche, Polymeric Gas Separation Membranes, Wiley, New York, 1993, pp. 77-91.

[23] B. Kunst, D. Skevin, G. Dezelic and J.J. Petres, A lightscattering and membrane formation study on concentrated cellulose acetate solutions, J. Appl. Polym. Sci., 20 (1976) 1339.

[24] K. Vásárhelyi, J.A. Ronner, M.H.V. Mulder and C.A. Smolders, Development of wet-dry reversible reverse osmosis membranes with high performance from cellulose acetate and cellulose triacetate blends, Desalination, 61 (1987) 211.

[25] J.A. Ronner, S. Groot Wassink and C.A. Smolders, Investigation of liquid-liquid demixing and aggregate formation in a membrane-forming system by means of pulse-induced critical scattering (PICS), J. Membrane Sci., 42 (1989) 27.

[26] M.A.M. Beerlage, Polyimide ultrafiltration membranes for non-aqeous systems, $\mathrm{PhD}$ thesis University of Twente, Enschede, 1994, chapter 3.

[27] E. Smit, Modelling of the diffusion of gases through membranes of novel polyimides, $\mathrm{PhD}$ thesis University of Twente, Enschede, 1991.

[28] M. Panar, H.H. Hoehn and R.R. Hebert, The nature of asymmetry in reverse osmosis membranes, Macromolecules, 6 (1973) 777.

[29] R.E. Kesting, Concerning the microstructure of dry RO membranes, J. Appl. Polym. Sci., 17 (1973) 1771.

[30] K. Kamide and S.I. Manabe, Role of microphase separation phenomena in the formation of porous polymeric membranes, ACS Symp. Ser., 269 (1985) 197.

[31] (a) B. Kunst and Z. Vajnaht, On the structure of concentrated cellulose acetate solutions, J. Appl. Polym. Sci., 21 (1977) 2505. (b) B. Kunst, D. Skevin and, G. Dezelic, A lightscattering and membrane formation study on concentrated cellulose acetate solutions, J. Appl. Polym. Sci., 20 (1976) 1339.

[32] R.J. Ray, W.B. Krantz, R.L. Sani, Linear stability theory model for finger formation in asymmetric membranes, J. Membrane Sci., 23 (1985) 155.

[33] K. Kimmerle and H. Strathmann, Analysis of the structuredetermining process of phase inversion membranes, Desalination, 79 (1990) 283.

[34] (a) 1. Pinnau, Skin formation of integral-asymmetric gas separation membranes made by dry/wet phase inversion, PhD thesis University of Texas, Austin, 1991. (b) I. Pinnau and W.J. Koros, J. Polym. Sci., Phys. Ed., 31 (1993) 419.

[35] R.M. Boom, I.M. Wienk, Th. v.d. Boomgaard and C.A. Smolders, Microstructures in phase inversion membranes. II. The role of a polymeric additive, J. Membrane Sci., 73 (1992) 277.

[36] I.M. Wienk, Th. van den Boomgaard and C.A. Smolders, The formation of nodular structures in the top layer of ultrafiltration membranes, J. Appl. Polym. Sci., accepted. 
[37] (a) N.K. Ailawadi and J. Naghizadeh, Collapse transition in polymers, Mol. Cryst. Liq. Cryst., 38 (1977) 171. (b) P.G. de Gennes, Kinetics of collapse for a flexible coil, J. Phys. Lett., 46 (1985) L639. (c) J. Yu, Z. Wang and B. Chu, Kinetic study of coil-to-globule transition, Macromolecules, 25 (1992) 1618.
[38] M.A.M. Beerlage, Polyimide ultrafiltration membranes for non-aqeous systems, PhD thesis University of Twente, Enschede, 1994, Appendix chapter 4.

[39] A.M.W. Bulte, Nylon-4,6 as membrane material; Polymer crystallization during immersion precipitation, $\mathrm{PhD}$ thesis University of Twente, Enschede, 1994. 